\title{
A beamforming study of the linear antenna array using grey wolf optimization algorithm
}

\author{
Asma Issa Mohsin ${ }^{1}$, Asaad. S. Daghal ${ }^{2}$, Adheed Hasan Sallomi ${ }^{3}$ \\ ${ }^{1}$ Ministry of Oil, Iraq \\ ${ }^{1,2}$ Al-Furat Al-Awsat Technical University, Iraq \\ ${ }^{3}$ Faculty of Engineering, Al-Mustansiriyah University, Iraq
}

\begin{tabular}{l} 
Article Info \\
\hline Article history: \\
Received Apr 14, 2020 \\
Revised Jun 4, 2020 \\
Accepted Jul 20, 2020 \\
\hline
\end{tabular}

\section{Keywords:}

Beamforming

Genetic algorithm

Grey wolf optimization

Smart antenna

\begin{abstract}
The grey wolf optimization (GWO) algorithm is considered an inspired meta-heuristic algorithm, which inspired by the social hierarchy and hunting behavior of the grey wolves. GWO has a high-performance capability of solving constrained, as well as unconstrained optimization problems. In this paper, the beamforming of smart antennas in a code division multiple access system based on the GWO algorithm is investigated. The sidelobe level (SLL) is minimized along with peak sidelobe level reduction, as well as an optimal beam pattern has been accomplished by using GWO to uniform linear antenna arrays. In this work, an amplitude is introduced as constant, while the interspacing distance between antenna array elements and the number of elements in a linear array are variables. The simulation results show that a faster convergence and likely high accurate beamforming are gained using GWO based method. Finally, it is shown that the GWO outperforms the genetic algorithm (GA) based method.
\end{abstract}

Copyright $@ 2020$ Institute of Advanced Engineering and Science. All rights reserved.

Corresponding Author:

Asaad. S. Daghal,

Department of Avionics Engineering,

Engineering Technical College-Najaf,

Al-Furat Al-Awsat Technical University, Kufa, Iraq.

Email: ad466kent@atu.edu.iq

\section{INTRODUCTION}

The demand for mobile communication services, such as voice and high-speed data rates is increased drastically during the last decade. Therefore, the mobile network operators are giving a significant priority to support more users per base station (BS) and provide a high quality of services at a low cost [1-3]. Since the capacity of the spectrum is limited due to the available transmission bandwidth is finite, attempts to boost traffic with a constant bandwidth produce more interference in the system and harmful the signal quality [4-6]. However, when using omnidirectional antennas at the base station, the transmitted and received signal of each user turns into a source of interference to other users, which lies in the same cell, making the overall system interference limited [7-9].

The main purpose of beamforming is used to steer multiple beams towards wanted users while cancelling the interferers at the same time [10-12]. It can do that through adjustment of the beamformers weight vectors, where the quality of the communication channel can maximize through the process of altering the complex weight [13-17]. To accomplish a narrow beam with high gain, a large number of antenna elements are compact together to construct the array [18-22]. Phased array antennas can steer the beam pattern electronically in particular directions, minimal SLL, and suppress interference [23-26]. However, the grey wolf optimization (GWO) algorithm was suggested by Mirjalili.et.al in 2014 [5], which inspired by the leadership pyramidal and hunting mechanism of the grey wolves. GWO is considered a 
promising algorithm for solving practical trouble situations in different engineering fields, such as welded beam design, optical buffer design, etc [5]. Therefore, it motivates us to use this algorithm (GWO) in antenna array to reinforce the performance of the system. The main contributions of this paper are:

a) The directivity has been increased significantly when a GWO is used compared to genetic algorithm (GA), which gives a sharper and more precise beam pattern.

b) The results show that convergence of a GWO is achieved faster than GA based method.

The rest of this paper is organized as follows. A geometry configuration of an antenna linear array and the equations of array factors are discussed in Section 2. The GWO algorithm and its implementation steps are described in Section 3. The array patterns are demonstrated in Section 4, as well as several examples of the linear array analysis along with the GWO optimized antenna currents and positions are presented. While the conclusion presents in Section 5.

\section{CONFIGURATION OF THE ANTENNA LINEAR ARRAY.}

The $N$-element array is considered a common general linear array. For simplicity, all elements in this study are assumed that have equal amplitudes and equally spaced. Figure 1 depicts a linear array of an N-element, which collected isotropic radiation of antenna elements. It is supposed that the $n^{\text {th }}$ element steers the $(N-1)$ elements via an electrical phase shift $\partial$ radians, where the phase shift can be easily performed through the phase shifting of the antenna current for each element. By supposing $r \gg d$ that represents the far-field conditions, a far-field distance is given by.

$$
d=2 D^{2} / \lambda
$$

where $r$ is the distance from element $n$ to far-field point and $D$ is the maximum antenna dimension. The array factor is defined as [1].

$$
A F=1+e^{j(k d \sin \emptyset+\partial)}+e^{j 2(k d \sin \emptyset+\partial)}+e^{j 3(k d \sin \emptyset+\partial)}+\cdots+e^{j(N-1)(k d \sin \emptyset+\partial)}
$$

where $\partial$ is the phase shift between successive elements. (2) can more precisely express as:

$$
A F=\sum_{n=1}^{N} e^{j(N-1)(\psi)}
$$

where $\psi=k d \sin \emptyset+\partial$.

It is noted that if the array is lined up along the $z-$ axis then $\psi=k d \cos \emptyset+\partial$

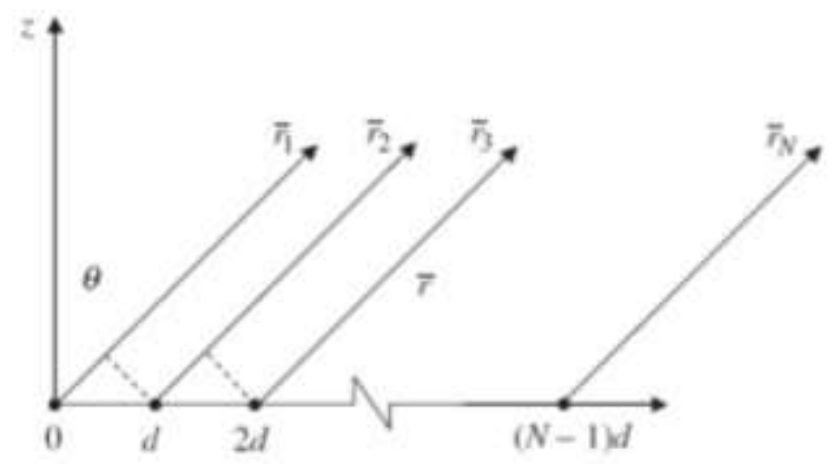

Figure 1. Uniform linear array geometry

\section{GREY WOLF OPTIMIZATION ALGORTHIM}

The GWO algorithm is considered one of the meta-heuristic algorithms that emulate the social hunting manner and hierarchical of the grey wolves group. The hunting manner of the grey wolves can be summarized as follows:-The first grade, which represents the chi $(\chi)$ that they are the leaders and can be males or females. The second grade in the hierarchical is consists of the rho $(\rho)$ wolves, which followed by the wolves called gamma $(\gamma)$, while the down sorting of the grey wolves is named mu $(\mu)$. The hierarchical of the grey wolves are constituted mathematically throughout counting $\chi$ as the best solution that followed by 
the $2^{\text {nd }}$ and the $3^{\text {rd }}$ finest solutions $\rho$ and $\gamma$, respectively. The remaining of the nominated solutions are supposed to be $\mu$. The major steps sequence of the grey wolf hunting can be summarized as follows $[5,6]$ :

a) Pursuit, chasing after and draw near the prey.

b) Keep tracking, surrounding, and beset the prey until it stops moving.

c) Raid towards the prey.

During a hunting process, grey wolves hedge the prey that is mathematically modelled as [5]:

$$
\begin{aligned}
& \vec{D}=|\vec{C} \cdot \overrightarrow{Y p(t)}-\overrightarrow{Y(t)}| \\
& \overrightarrow{y(t+1)}=\overrightarrow{Y p(t)}-\vec{A} \cdot \overrightarrow{(D)}
\end{aligned}
$$

where $\vec{C}$ and $\vec{A}$ are the coefficient vectors, $t$ is referred to the current iteration, $\overrightarrow{Y p}$ represents the location vector of the prey, and $\vec{Y}$ refers to the location vector of the grey wolf. The vectors $\vec{C}$ and $\vec{A}$ are evaluated as:-

$$
\begin{aligned}
\vec{C} & =2 \cdot \overrightarrow{r_{2}}, \\
\vec{A} & =2 \vec{\chi} \cdot \overrightarrow{r_{1}}-\vec{\chi},
\end{aligned}
$$

where components of $\vec{\chi}$ are decreased linearly from 2 to 0 during the iterations and $\overrightarrow{r_{1}}, \overrightarrow{r_{2}}$ are random vectors that lie in the range $[0,1]$. In the GWO algorithm process, the hunting approach has led by $\chi, \rho$, and $\gamma$, where the other wolves follow those three types of wolves. Further, in the pack of the wolves, it is supposed that the wolves' $\chi, \rho$, and $\gamma$ have the best familiarity about the potential position of the prey. Consequently, the first three best solutions will be save, and the other searching operators will update their locations according to the position of the best search operator. For this purpose, we use the following [5]:

$$
\left.\begin{array}{c}
\overrightarrow{D \chi}=|\overrightarrow{C 1} \cdot \overrightarrow{Y \chi}-\vec{Y}| \\
\overrightarrow{D \rho}=|\overrightarrow{C 2} \cdot \overrightarrow{Y \rho}-\vec{Y}| \\
\overrightarrow{D \gamma}=|\overrightarrow{C 3} \cdot \overrightarrow{Y \gamma}-\vec{Y}| \\
\overrightarrow{Y 1}=\overrightarrow{Y \chi}-\overrightarrow{A 1} \cdot \overrightarrow{(D \chi)} \\
\overrightarrow{Y 2}=\overrightarrow{Y \rho}-\overrightarrow{A 2} \cdot \overrightarrow{(D \rho)} \\
\overrightarrow{Y 3}=\overrightarrow{Y \gamma}-\overrightarrow{A 3} \cdot \overrightarrow{(D \gamma)} \\
\vec{Y}(t+1)=\frac{\overrightarrow{Y 1}+\overrightarrow{Y 2}+\overrightarrow{Y 3}}{3}
\end{array}\right\}
$$

By using (8), and according to the parameters $\chi, \rho$, and $\gamma$ in the nth-dimensional searching domain, which is depicted in Figure 2, a search operator will be updating its location [5].

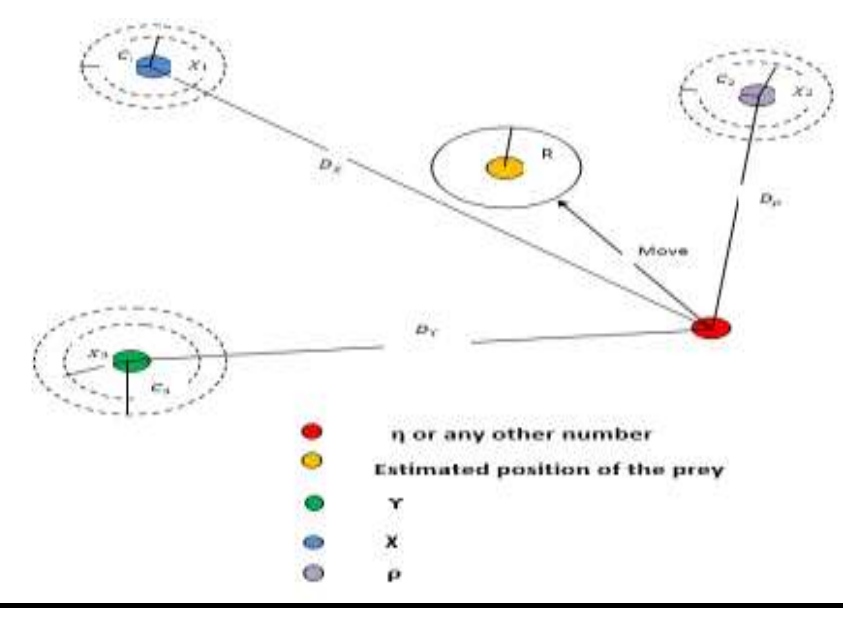

Figure 2. Location update in GWO 
Also, the final location of the search domain would be in a random place within a circle that is clarified by the locations of $\chi, \rho$, and $\gamma$. So that, $\chi \rho \rho$, and $\gamma$ evaluate the location of the prey, while the rest of the wolves update their locations randomly throughout the prey [5-7]. However, the GWO algorithm is summarized as follow [5,6, 8, 9]:

a) Make a random population of grey wolves (candidate solutions).

b) $\chi, \rho$, and $\gamma$ wolves evaluate the likely position of the prey throughout iterations, where each candidate updates its distance according to the prey position.

c) The factor " $\vec{\chi}$ " is linearly reduced from 2 to 0 , in order to confirm the searching and raiding of the prey, respectively. To avoid recession in local solutions, the candidate solutions will tend to converge towards the prey when $|\vec{A}|<1$ and diverge from the prey when $|\vec{A}|>1$ and $[5,11]$.

d) Eventually, the GWO algorithm is stopped by the contentment of an end criterion.

\section{RESULTS AND ANALYSIS}

A GWO technique is utilized to reduce the SLL, as long as to direct the main beam at different angles. Simulation tool of MATLAB software has been used to analyze the performance of the system, where a curve between the SLL and the phase angle of the different elements has taken into consideration. Besides, simulation results were taken in both GA and GWO techniques for various array antenna designs. The main simulation parameters of this paper are shown in Table 1.

Table 1. Simulation parameters

\begin{tabular}{cc}
\hline Parameter & Description \\
\hline Direction of arrival (DOA) angle & DOA1 $=30 \mathrm{o}$, DOA2 $=-450$ \\
Frequency & $900 \mathrm{MHZ}$ \\
Element spacing & $0.25 \lambda, 0.5 \lambda, 0.75 \lambda, 1 \lambda, 2 \lambda$ \\
No of iteration for GWO & 5 \\
\hline
\end{tabular}

In our investigation, there is an array of 4,8,16 elements with the possibility of changing the distance between elements by $0.25 \lambda, 0.5 \lambda, 0.75 \lambda, 1 \lambda$ and $2 \lambda$. In Table 2 , an average of all antenna array parameters for the case study has been taken, where all study cases of inter-element spacing, according to the number of elements, which are 4, 8 and 16. As we notice the increasing number of antennas in the array will result in increasing of D1 and D2, which means greater directivity. Further, the half-power beamwidth (HPBW), HPBW1 and HPBW2 are decreasing that means more accurate and sharper beam pattern, although this improvement brings greater SLL and more side lobe numbers.

Table 3 shows the average of the same parameters according to inter-element distances for all three cases of elements numbers. As we can recognize the increasing of inter-element spacing, will results in grater HPBW that means less precise beam pattern. This increase will perform greater SLL and more numbers of side lobes. Tables 4 and 5 represent the average of parameters according to number of elements and the average of parameters according to inter-spacing distance for same case studies simulated by GA algorithm. In Table 4, we can notice an increase in the number of samples needed for convergence by increasing the number of elements this means that we will need more processing time while the number of samples and processing time in the GWO algorithm is same for all number of elements. In Table 6, two examples as a comparison between beam pattern and HPBW and other parameters achieved by grey wolf optimization technique and genetic algorithm are considered.

In the first case, a 4-elements antenna array with $0.25 \lambda$ inter-spacing between elements has been studied. Figure 3 (a, b, c, and d) and Figure 4 (a, b, c and d) show that the curves of parameters which achieved by applying the GWO and GA algorithm, respectively. It is clear from rapprochement in Table 6 that the directivity of DOA 1 and DOA 2 are increased by 0.69 and 0.73 degrees, respectively, when the GWO technique is used, which in turn results in a sharper and more precise beam pattern. However, the number of SLL in both optimization techniques remains one for each DOA. Furthermore, Figure $3(\mathrm{a} \& \mathrm{~b})$ and Figure 4 (a \& b) illustrated that the applying GA, results in less SLL. In addition, from Figure 3 (c \& d) and Figure 4 (c \& d), it can be noticed that using the GA algorithm can reduce the SLL into $11 \mathrm{~dB}$ and $13 \mathrm{~dB}$ for first and second DOA respectively which means 12.58 and 19.95 watts less power in comparison with the use of GWO. In GA, the convergence process was achieved after 30 iterations, while it has taken five iterations in the GWO technique.

Table 2. The average of parameters according to number of elements for GWO

\begin{tabular}{cccccccccc}
\hline Item & No.Element & D1 & D2 & HPBW1 & HPBW2 & SLL1 & SLL2 & No.SL1 & NO.SL2 \\
\hline 1 & 4 & 5.246 & 5.128 & 43.4 & 41 & 0.3 & 0.28 & 1 & 1 \\
2 & 8 & 8.33 & 8.26 & 19.2 & 23.6 & 0.28 & 0.29 & 3 & 2 \\
3 & 16 & 10.97 & 10.87 & 10.4 & 9.2 & 0.27 & 0.32 & 4 & 5 \\
\hline
\end{tabular}


Table 3. The average of parameters according to inter-spacing distance for GWO

\begin{tabular}{cccccccccc}
\hline Item & Distance & D1 & D2 & HPBW1 & HPBW2 & SLL1 & SLL2 & No.SL1 & NO.SL2 \\
\hline 1 & 0.25 & 8.44 & 8.03 & 20.66 & 22 & 0.3 & 0.28 & 2 & 2 \\
2 & 0.5 & 7.99 & 8.04 & 24 & 23.66 & 0.30 & 0.41 & 3 \\
3 & 0.75 & 8.05 & 7.96 & 24.66 & 30.66 & 0.28 & 0.33 & 2 \\
4 & 1 & 8.26 & 8.16 & 25.33 & 22.66 & 0.29 & 0.26 & 3 \\
5 & 2 & 8.2 & 8.02 & 24.33 & 24 & 0.227 & 0.22 & 3 & 2 \\
\hline
\end{tabular}

Table 4. The average of parameters according to number of elements for GA

\begin{tabular}{cccccccccccc}
\hline Item & No.Element & D1 & D2 & HPBW1 & HPBW2 & SLL1 & SLL2 & No.SL1 & NO.SL2 & Converg. \\
\hline 1 & 4 & 5.372 & 5.256 & 43.8 & 46.2 & 0.149 & 0.152 & 0 & 0 & 65 \\
2 & 8 & 7.86 & 7.82 & 23.4 & 22.6 & 0.184 & 0.178 & 1 & 1 & 101 \\
3 & 16 & 11.136 & 11.084 & 10 & 10.8 & 0.194 & 0.148 & 4 & 4 & 134 \\
\hline
\end{tabular}

Table 5. The average of parameters according to inter-spacing distance for GA

\begin{tabular}{ccccccccccc}
\hline Item & Distance & D1 & D2 & HPBW1 & HPBW2 & SLL1 & SLL2 & No.SL1 & NO.SL2 & Convergence \\
\hline 1 & 0.25 & 8.17 & 8.13 & 24 & 27.33 & 0.23 & 0.17 & 2 & 2 & 93 \\
2 & 0.5 & 8.24 & 8.19 & 27 & 26.33 & 0.13 & 0.11 & 2 & 1 & 2 \\
3 & 0.75 & 7.92 & 8.07 & 25.33 & 27.33 & 0.19 & 0.13 & 2 & 2 \\
4 & 1 & 8.15 & 8.21 & 25.33 & 24.66 & 0.16 & 0.18 & 2 & 2 & 61 \\
5 & 2 & 8.12 & 7.74 & 27 & 27 & 0.15 & 0.18 & 2 & 2 & 193 \\
\hline
\end{tabular}

Table 6. Comparison between two considered cases parameters

\begin{tabular}{cccccccccccc}
\hline Item & $\mathrm{N}$ & $\lambda$ & Algorithm & $\mathrm{D} 1$ & $\mathrm{D} 2$ & HPBW1 & HPBW2 & SLL1 & SLL2 & No.SL1 & NO.SL2 \\
\hline 1 & 4 & 0.25 & GWO & 6.02 & 5.32 & 36 & 38 & 0.23 & 0.35 & 1 & 1 \\
2 & 4 & 0.25 & GA & 5.33 & 4.59 & 40 & 54 & 0.07 & 1.5 & 1 & 1 \\
3 & 16 & 1 & GWO & 11.29 & 10.88 & 10 & 8 & 0.21 & 0.2 & 4 & 4 \\
4 & 16 & 1 & GA & 10.91 & 11.08 & 10 & 10 & 0.18 & 0.11 & 4 & 4 \\
\hline
\end{tabular}

In the second case, the16-element antenna array with $1 \lambda$ interspacing between elements is considered. Figure 5 (a, b, c, and d) and Figure 6 (a, b, c, and d) show the curves generated by applying GWO and GA, respectively. From the results of Table 6, it can be observed that the directivity of DOA1 is increased by 0.38 degrees when the GWO technique is used compared with the GA. However, the HPBW decreases by 4 and 6 degrees for the first and second DOA, respectively. Figure 5 (a \& b) and Figure 6 (a \& b) shows that applying GA results in less SLL by 0.03 and 0.09 for $1^{\text {st }}$ and $2^{\text {nd }}$ DOA, respectively.

From the Figure 5 (c, d) and Figure 6 (c, d), it can easily conclude that using the GA provides us 10 and $3 \mathrm{~dB}$ in the first and the second DOA, respectively, which equals to 10 and 1.99 watts less power in comparison with the using of GWO algorithm. In contrast, the sidelobe levels run high by 0.1 and 0.23 for the $1^{\text {st }}$ and $2^{\text {nd }}$ DOA in the GWO technique. Besides, the convergence in GA takes 110 iterations, while it uses only five iterations in the GWO technique.

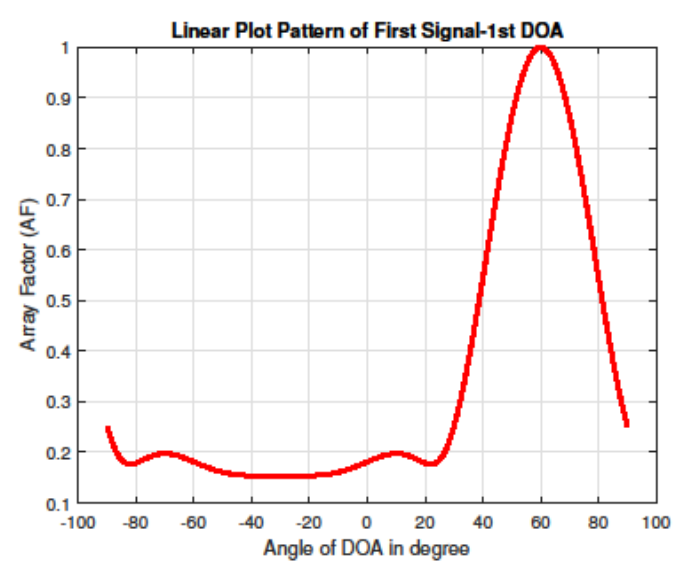

Figure 3(a). SLL, main lobe of $1^{\text {st }} \mathrm{DOA}$ for $\mathrm{N}=4$, $\lambda=0.25, \mathrm{GWO}$

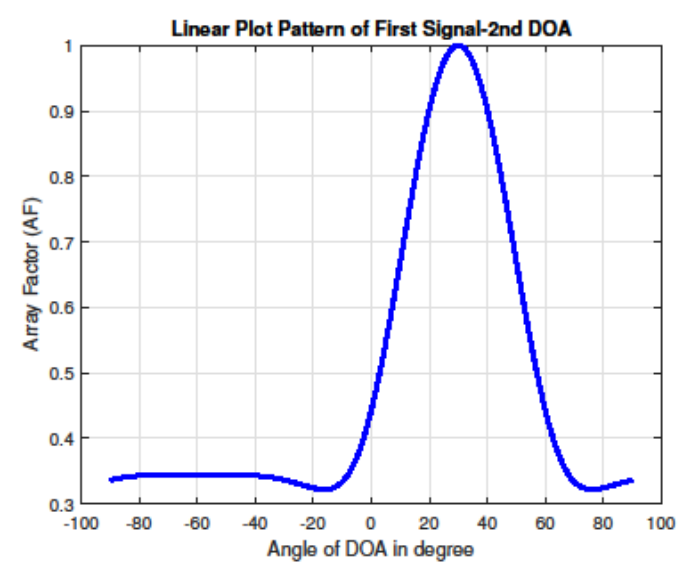

Figure 3(b). SLL, main lobe of $2^{\text {nd }}$ DOA for $\mathrm{N}=4$, $\lambda=0.25, \mathrm{GW}$ 


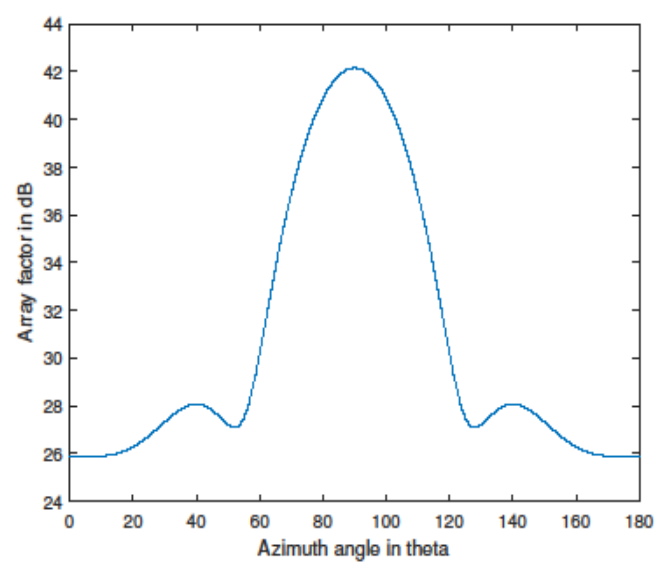

Figure 3(c). SLL of $1^{\text {st }} \mathrm{DOA}$ in $\mathrm{dB}$ for $\mathrm{N}=4$, $\lambda=0.25, \mathrm{GWO}$

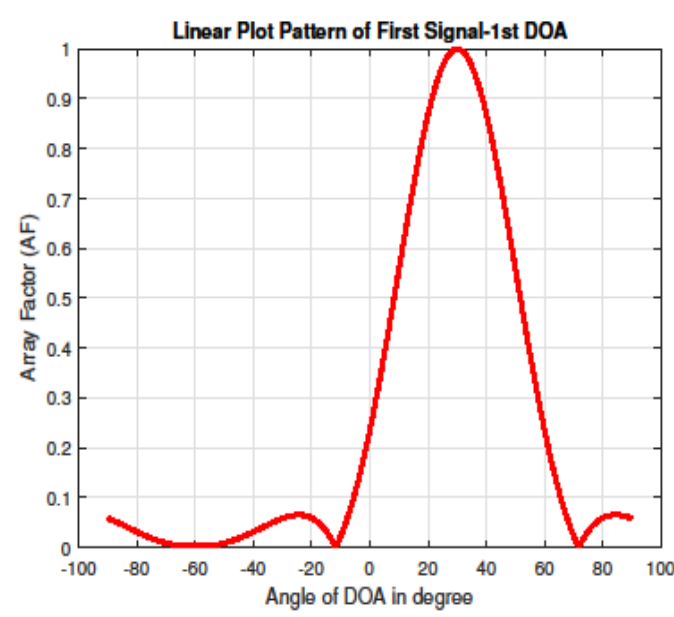

Figure 4(a). SLL, main lobe of $1^{\text {st }}$ DOA for $\mathrm{N}=4$, $\lambda=0.25, \mathrm{GA}$

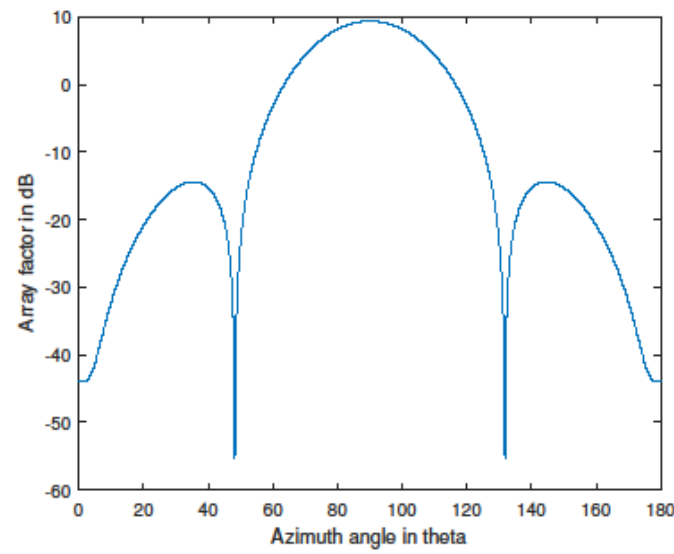

Figure 4(c). SLL of $1^{\text {st }}$ DOA in $\mathrm{dB}$ for $\mathrm{N}=4$, $\lambda=0.25, \mathrm{GA}$

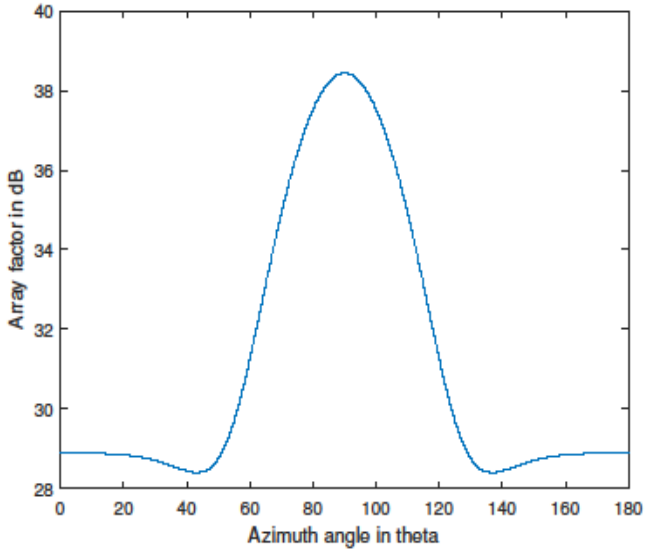

Figure 3(d). SLL of $2^{\text {nd }} D O A$ in $d B$ for $N=4$, $\lambda=0.25$, GWO

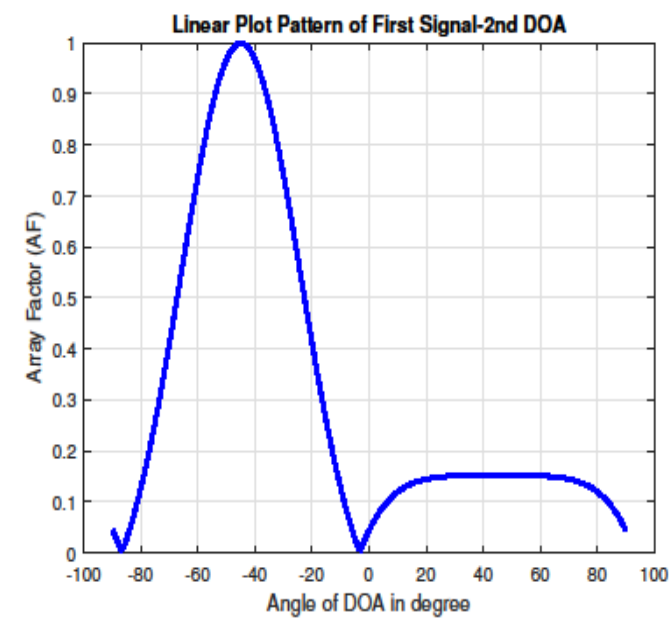

Figure 4(b). SLL, main lobe of $2^{\text {nd }}$ DOA for $N=4$, $\lambda=0.25, \mathrm{GA}$

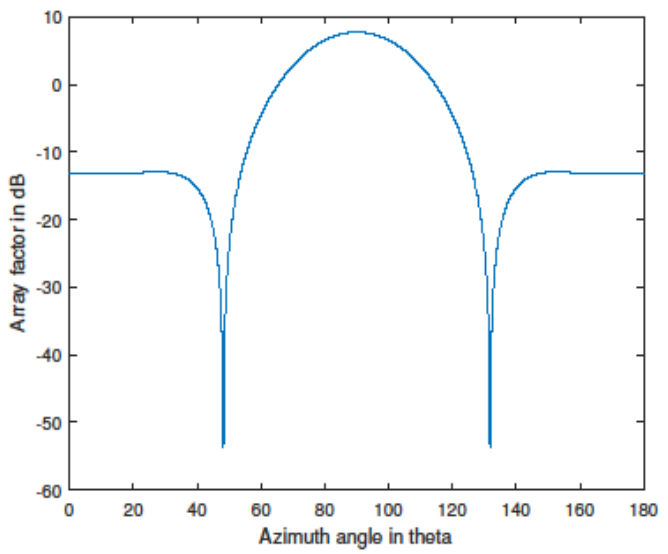

Figure 4(d). SLL of $2^{\text {nd }}$ DOA in $d B$ for $N=4$, $\lambda=0.25, \mathrm{GA}$ 


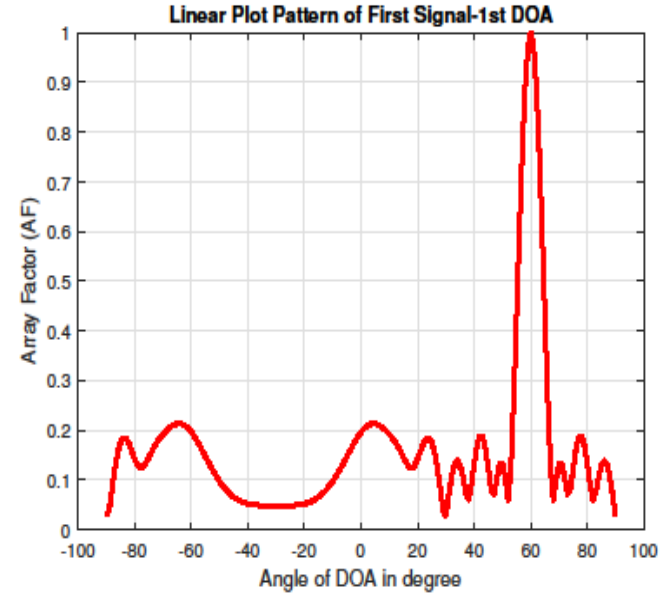

Figure 5(a). SLL, main lobe of $1^{\text {st }} \mathrm{DOA}$ for $\mathrm{N}=16$, $\lambda=0.25, \mathrm{GW}$

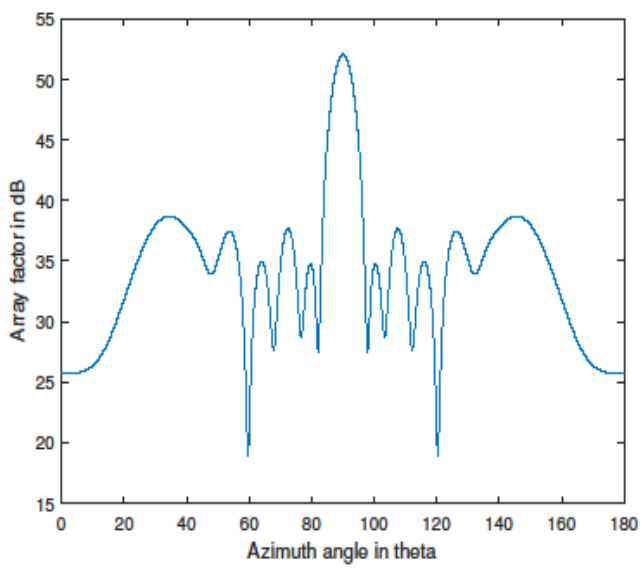

Figure 5(c). SLL of $1^{\text {st }}$ DOA in $\mathrm{dB}$ for $\mathrm{N}=16$, $\lambda=0.25, \mathrm{GWO}$

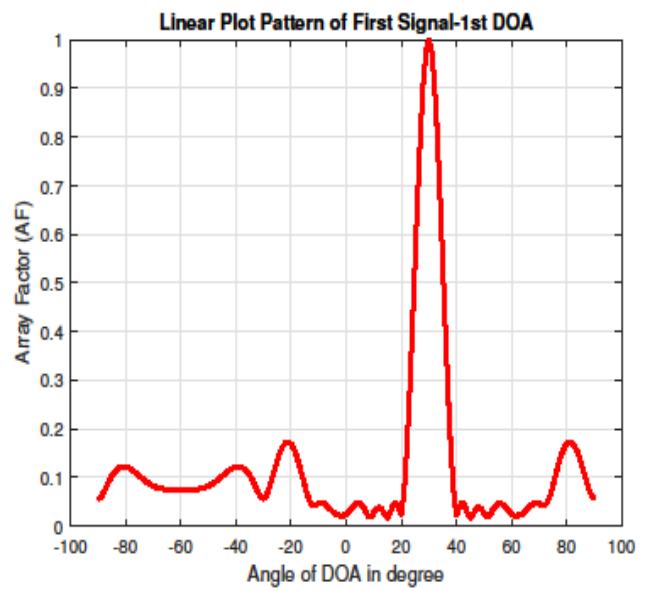

Figure 6(a). SLL, main lobe of $1^{\text {st }}$ DOA for $\mathrm{N}=16$, $\lambda=0.25, \mathrm{GA}$

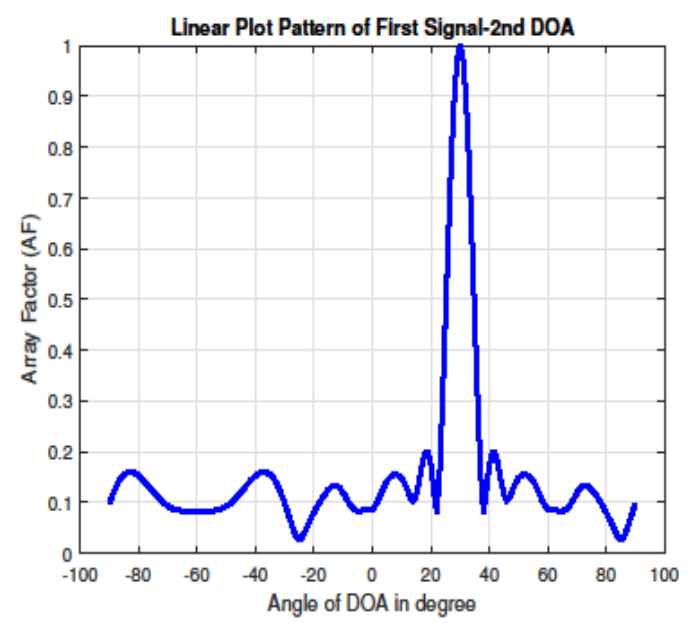

Figure 5(b). SLL, main lobe of $2^{\text {nd }} \mathrm{DOA}$ for $\mathrm{N}=16$, $\lambda=0.25$, GWO

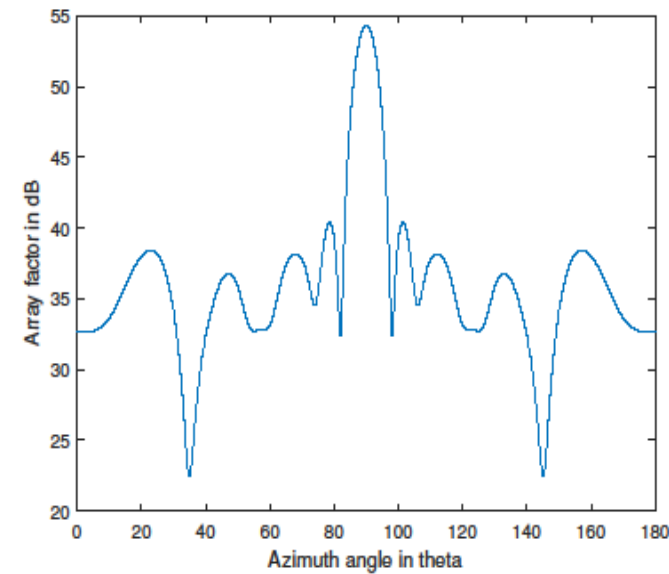

Figure 5(d). SLL of $2^{\text {nd }}$ DOA in $d B$ for $\mathrm{N}=16$, $\lambda=0.25$, GWO

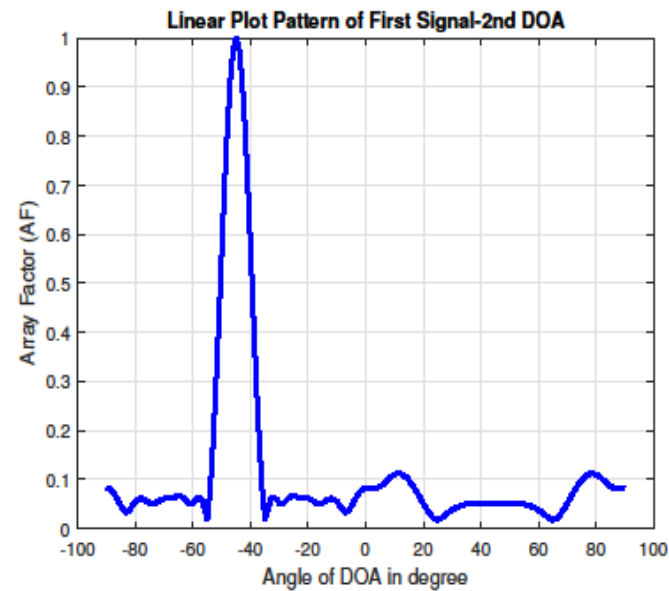

Figure 6(b). SLL, main lobe of $2^{\text {nd }}$ DOA for $N=16$, $\lambda=0.25, \mathrm{GA}$ 


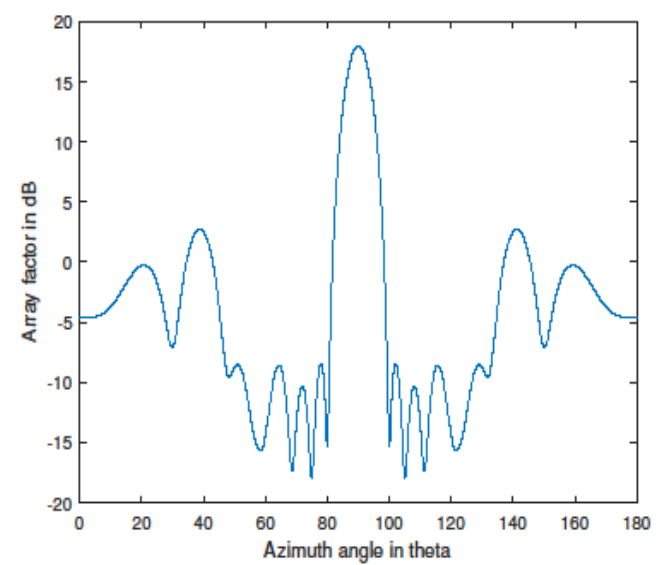

Figure 6(c). SLL of 1 st DOA in $\mathrm{dB}$ for $\mathrm{N}=16$, $\lambda=0.25, \mathrm{GA}$

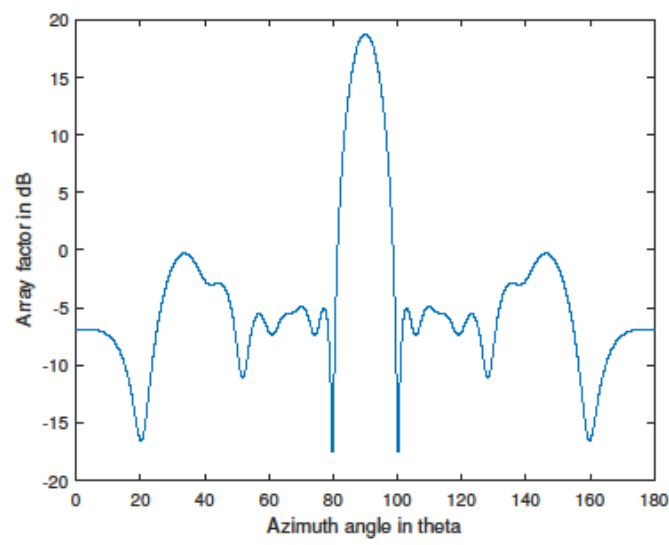

Figure 6(d). SLL of 2nd DOA in $\mathrm{dB}$ for $\mathrm{N}=16$, $\lambda=0.25, \mathrm{GA}$

\section{CONCLUSION}

In this paper, the GWO algorithm has studied as an optimization technique for beamforming to achieve optimal beam pattern with maximum sidelobe reduction for smart linear arrays. In general, by using both algorithms we can recognize that an increase in the number of elements in antenna array led to better beam patterns. This improvement is at the expense of sidelobe numbers and sidelobe level growth. It can also observe that the increase of the distance between elements can result in better directivity and narrower beamwidth on the account of more side lobes numbers. The findings of GWO optimization algorithm have been compared with the GA optimization technique results. The findings demonstrated that GWO provides significant enhancements in beamforming and the results are generally close to outcomes from GA algorithm. It concludes that the use of GWO optimization technique improves the directivity of the smart antenna and needs fewer samples sequentially less computation time in rapprochement with GA.

\section{REFERENCES}

[1] C. A. Balanis, "Antenna theory: analysis and design," John wiley \& sons, 2015.

[2] D.-C. Chang and C.-N. Hu, "Smart antennas for advanced communication systems," Proceedings of the IEEE, vol. 100 , no. 7 , pp. 2233-2249, 2012.

[3] M. Agiwal, A. Roy, and N. Saxena, "Next generation $5 \mathrm{~g}$ wireless networks: A comprehensive survey," IEEE Communications Surveys \& Tutorials, vol. 18, no. 3, pp. 1617-1655, 2016.

[4] S. F. Maharimi, et al., "Impact of spacing and number of elements on array factor," Proc. of Progress in Electromagnetics Research Symposium, 2012.

[5] S. Mirjalili, S. M. Mirjalili, A. Lewis, "Grey Wolf Optimizer," In advances in engineering software, vol. 69, pp. 46-61, 2014.

[6] A.A.El-Gaafary,et al.,"Grey wolf optimization for multi input multi output system," Universal Journal of Communications and Network, vol. 3, no. 1, pp. 1-6, 2015.

[7] H. M. Song, M. H. Sulaiman, and M. R. Mohamed, "An application of grey wolf optimizer for solving combined economic emission dispatch problems," International Review on Modelling and Simulations, vol. 7, no. 5, pp. 838-844, 2014.

[8] L. I. Wong, et al., "Grey Wolf Optimizer for solving economic dispatch problems," 2014 IEEE International Conference on Power and Energy (PECon), Kuching, pp. 150-154, 2014. doi: 10.1109/PECON.2014.7062431.

[9] C. Muro, et a., "Wolf-pack (canis lupus) hunting strategies emerge from simple rules in computational simulations," Behavioral processes, vol. 88, no. 3, pp. 192-197, 2011.

[10] R. A. Hasan, et al., "A comprehensive study: Ant Colony Optimization (ACO) for facility layout problem," in 2017 16th RoEduNet Conference: Networking in Education and Research (RoEduNet), pp. 1-8, 2017.

[11] R. A. Hasan and M. N. Mohammed, "A krill herd behaviour inspired load balancing of tasks in cloud computing," Studies in Informatics and Control, vol. 26, no 4, pp. 413-424, 2017.

[12] Z. F. Hussain, et al., "A new model for iris data set classification based on linear support vector machine parameter's optimization," International Journal of Electrical \& Computer Engineering, vol. 10, no. 1, pp. 1079-1084, 2020.

[13] M. A. Mohammed and R. A. Hasan, "Particle swarm optimization for facility layout problems FLP-A comprehensive study," in 2017 13th IEEE International Conference on Intelligent Computer Communication and Processing (ICCP), pp. 93-99, 2017. 
[14] M. A. Mohammed, et al., "A Focal load balancer based algorithm for task assignment in cloud environment," in 2018 10th International Conference on Electronics, Computers and Artificial Intelligence (ECAI), pp. 1-4, 2018.

[15] M. A. Mohammed, et al., "An Effective Context Sensitive Offloading System for Mobile Cloud Environments using Support Value-based Classification," Scalable Computing: Practice and Experience, vol. 20, no. 4, pp. 687-698, 2019.

[16] M. A. Mohammed, et al., "Green Energy Sources: Issues and Challenges," in 2019 18th RoEduNet Conference: Networking in Education and Research (RoEduNet), pp. 1-8, 2019.

[17] M. A. Mohammed, et al., "Security and accountability for sharing the data stored in the cloud," in 2016 15th RoEduNet Conference: Networking in Education and Research, pp. 1-5, 2016.

[18] M. A. Mohammed and N. ŢĂPUŞ, "A Novel Approach of Reducing Energy Consumption by Utilizing Enthalpy in Mobile Cloud Computing," Studies in Informatics and Control, vol. 26, no. 4, pp. 425-434, 2017.

[19] N. Q. Mohammed, et al., "Comparative Analysis between Solar and Wind Turbine Energy Sources in IoT Based on Economical and Efficiency Considerations," in 2019 22nd International Conference on Control Systems and Computer Science (CSCS), pp. 448-452, 2019.

[20] M. A. A. Royida et al., "Efficient method for breast cancer classification based on ensemble hoffeding tree and naïve Bayes," Indonesian Journal of Electrical Engineering and Computer Science, vol. 18, no. 2, pp. 1074-1080, 2020.

[21] Z. H. Salih, G. T. Hasan, and M. A. Mohammed, "Investigate and analyze the levels of electromagnetic radiations emitted from underground power cables extended in modern cities," in 2017 9th International Conference on Electronics, Computers and Artificial Intelligence (ECAI), pp. 1-4, 2017.

[22] Z. H. Salih, et 1., "Study the Effect of Integrating the Solar Energy Source on Stability of Electrical Distribution System," in 2019 22nd International Conference on Control Systems and Computer Science (CSCS), pp. 443-447, 2019.

[23] N. D. Zaki, et al., "A real-time big data sentiment analysis for iraqi tweets using spark streaming," Bulletin of Electrical Engineering and Informatics, vol. 9, no 6, 2020.

[24] O. A. Hammood, et al., "The VANET-Solution Approach for Data Packet Forwarding Improvement," Advanced Science Letters, vol. 24, no 10, pp. 7423-7427, 2018.

[25] O. A. Hammood, et al., "RESP: Relay Suitability-based Routing Protocol for Video Streaming in Vehicular Ad Hoc Networks," International Journal of Computers, Communications \& Control, vol. 14, no 1, pp. 21-38, 2019.

[26] R. A. Hasan, et al., "HSO: A Hybrid Swarm Optimization Algorithm for Reducing Energy Consumption in the Cloudlets," Telkomnika, vol. 16, no. 5, pp. 2144-2154, 2018. 